\title{
Is Routine Audiometric Evaluation Necessary in Gynaecologic Tumour Patients Undergoing Chemotherapy?
}

\author{
Ayotunde J. Fasunla ${ }^{a, b} \quad$ Nadia Harbeck $^{c}$ Barbara Schmalfeld ${ }^{d}$ Sabina Berktold ${ }^{d}$ \\ Christina Böhner ${ }^{d}$ Walter Hundt ${ }^{\dagger} \quad$ Petra Wolf ${ }^{g}$ Silke Steinbach ${ }^{a}$ \\ aDepartment of Otorhinolaryngology, Philipps-University, Marburg, Germany, \\ ${ }^{b}$ Department of Otorhinolaryngology, College of Medicine, University of lbadan and University College Hospital, Ibadan, Nigeria, \\ 'Breast Centre, Ludwig-Maximilians University, Munich, \\ dDepartment of Obstetrics and Gynaecology, Women's Hospital Rechts der Isar, \\ fDepartment of Radiology, Philipps-University, Marburg, \\ IInstitute of Medical Statistics and Epidemiology, Hospital Rechts der Isar, Technical University Munich, Germany
}

\section{Keywords}

Breast cancer · Ovarian cancer - Chemotherapy . Hearing function

\section{Summary}

Background: Our objective was to assess the auditory function of gynaecological tumour patients who had received cytotoxic agents and to determine their associated risk of ototoxicity. Patients and Methods: 87 patients who had undergone chemotherapy for gynaecological malignancies were investigated. Of these patients, $79 \%$ had breast cancer, and $14 \%$ ovarian cancer. All of the patients had a subjective assessment of their hearing function on a visual analogue scale. Audiometric tests were performed before and at 9 weeks, 18 weeks and 3 months after completion of chemotherapy. Results: The age of the patients ranged from 32 to 71 years (mean age of $53.5 \pm 10.5$ years). The average subjective rating of the patients' hearing function was $83.0 \pm 17.2$ before and $84.8 \pm 16.93$ months after completion of chemotherapy. No significant audiometric change at either the speech hearing frequency range $(0.5-2 \mathrm{KHz})$ or high frequencies was observed in the patients after chemotherapy. There was also no significant difference in the hearing threshold of the patients who had received platinum analogue-based chemotherapy compared to non-platinum analogue-based chemotherapy. Conclusion: Hearing loss is uncommon in patients treated with the typical gynaecological chemotherapy protocols. Hence, routine audiometric testing in these patients is not necessary.
Schlüsselwörter

Mammakarzinom - Ovarialkarzinom - Chemotherapie · Hörfunktion

\section{Zusammenfassung}

Hintergrund: Ziel dieser Studie war es, die Hörfunktion von gynäkologischen Tumorpatientinnen unter den derzeit gängigen Chemotherapie-Schemata zu untersuchen und eine mögliche Ototoxizität der Chemotherapien abzuklären. Patientinnen und Methoden: Es wurden insgesamt 87 gynäkologische Tumorpatientinnen (darunter 79\% Mammakarzinom- und 14\% Ovarialkarzinom-Patientinnen) die mit derzeit gängigen Chemotherapie-Schemata behandelt wurden, untersucht. Das Hörvermögen wurde sowohl subjektiv auf einer visuellen Analogskala abgefragt als auch mittels Reintonaudiogramm vor Chemotherapie, 9 und 18 Wochen nach Beginn der Chemotherapie und 3 Monate nach Beendigung der Chemotherapie getestet. Ergebnisse: Die Patientinnen waren zwischen 32 und 71 Jahren alt (im Durchschnitt $53,5 \pm 10,5$ Jahre). Auf einer visuellen Analogskala ( $0=$ kein Hörvermögen, $100=$ exzellentes Hörvermögen) schätzten sich die Patientinnen im Durchschnitt mit einem Wert von $83,0 \pm 17,2$ vor Chemotherapie und $84,8 \pm 16,93$ Monate nach Beendigung der Chemotherapie ein. In den Reintonaudiogrammen zeigte sich kein signifikanter Unterschied in den Hörschwellenwerten im Sprach- als auch im Hochtonfrequenzbereich zu allen Messzeitpunkten. Es gab keinen signifikanten Unterschied in den Hörschwellenwerten von Patientinnen mit und ohne platinhaltiger Chemotherapie. Zusammenfassung: Ein Hörverlust bei gynäkologischen Tumorpatientinnen unter den derzeit gängigen Chemotherapie-Schemata ist unwahrscheinlich und es bedarf bei diesen Patientinnen keiner routinemäßigen audiometrischen Kontrolle.

\section{KARGER}

Fax +497614520714

Information@Karger.com

www.karger.com (c) 2013 S. Karger GmbH, Freiburg

$1661-3791 / 13 / 0084-0276 \$ 38.00 / 0$

Accessible online at:

www.karger.com/brc
Silke Steinbach, MD

Department of Otorhinolaryngology, Head and Neck Surgery

Philipps-University Marbur

Baldingerstraße, 35033 Marburg, Germany

silkesteinbach@hotmail.con 


\section{Introduction}

Cancer still accounts for more deaths than heart diseases in people younger than 85 years of age [1]. Breast cancer for example is the most common gynaecological malignancy diagnosed in both American and German women [2, 3]. However, progress has been made in reducing the incidence and mortality rate of the cancer disease, as well as improving the survival rate of these patients [1]. The better outcomes of this disease may be due to the availability of better equipment for the early detection and diagnosis of cancer. It may also be attributed to rapid changes in the recognization of risk factor patterns and the introduction of newer and better chemotherapeutic agents for treatments.

Chemotherapy is an effective treatment that may dramatically improve cancer symptoms and the overall survival rate of the cancer patients. However, there is still some uncertainty about whether the chemotherapy that is used to treat cancer patients can be employed in a way that ensures the preservation of the patients' hearing. The issue of quality of life - with respect to hearing - of patients undergoing chemotherapy is, therefore, an important issue.

Most gynaecological tumours are treated with surgery, followed by adjunct chemotherapy, with or without radiotherapy. The chemotherapeutic agents used in the treatment of these gynaecological patients are platinum-based and nonplatinum-based analogue agents. Platinum analogues such as cisplatin and carboplatin are effective antineoplastic agents. Unfortunately, various reports have associated these agents with toxicities, especially when high doses are administered or when the patients have been previously primed with chemotherapy. Cisplatin is the most ototoxic drug known $[4,5]$. High-dose carboplatin can also induce auditory dysfunction with a clinical picture that is roughly similar to cisplatinumcontaining chemotherapy $[6,7]$. The toxicity of these platinum-based agents has been demonstrated to be dose limiting $[7,8]$. It has also been shown to worsen the hearing threshold with a background disability of hearing loss [9]. Reports on the adverse effect of platinum analogues on the hearing function of women with gynaecological conditions are not common in the literature and are contradictory; some studies have reported no significant audiometric changes [10], whereas others described high-frequency hearing loss [4, 11].

There are few studies on the effect of other non-platinum analogue-based chemotherapeutic agents on hearing [12-14]. This study was, therefore, performed to assess the auditory function of gynaecological tumour patients who had received platinum analogue- and non-platinum analogue-based chemotherapeutic agents to determine whether the ototoxicity side effect of the chemotherapeutic agents were experienced by these patients. We also investigated the non-platinum-based chemotherapeutic agents mostly employed in gynaecological cancers for their auditory side effects.

\section{Patients and Methods}

Patients

This was a prospective hospital-based study of 87 consecutive patients who had undergone chemotherapy for gynaecological malignancies. Approval to conduct the study was obtained from Ethics Committee of the Faculty of Medicine at the Technical University of Munich in Germany (No. 1677/06). All the study patients gave written informed consent. The type of chemotherapy regimen received by individual patients was determined by the primary managing oncologist. There was no record of any patient being lost to follow-up; each patient participated in the study until 3 months after completion of the chemotherapy.

\section{Questionnaire}

A structured questionnaire was used to collect essential clinical data, which included the patient's age, menopause, tumour diagnosis, previous malignancy, previous chemotherapy and current surgical and/or chemotherapy regimen. Data on hearing loss, tinnitus, vertigo, family history of hearing loss, hearing aids, previous history of ear surgery, use of non-cytotoxic medication known to induce hearing loss and history of other medical diseases such as rhinosinusitis, otitis media, liver or renal diseases, hyperactivity or hypoactivity of the thyroid gland, diabetes mellitus, high blood pressure and neurological disorders were also collected.

\section{Subjective Assessment of Hearing Function}

The hearing function of each patient was subjectively assessed before chemotherapy and 3 months after its completion. A visual analogue scale (range 0-100) was used, where 0-20 represented very poor, 30-40 poor, 50-60 good, 70-80 very good and 90-100 excellent. Subjective assessments of vertigo, tinnitus and ear pressure were also performed on the same scale. The patients' ears were clinically examined before audiometric testing was performed; any ear wax found was removed.

\section{Audiometry}

Audiometric evaluations (threshold) were obtained for each ear at frequencies between 250 and 8,000 Hz. Measurements were taken using a pure tone-screening audiometer ST 3 (Audio-Med, Braunschweig, Germany). Both air and bone conduction tests were performed in a sound proof room separately on both ears. Each patient's hearing was tested 4 times: before ( 0 weeks) and during chemotherapy ( 9 weeks), and immediately after (18 weeks), and 3 months after ( 30 weeks) completion. The difference in hearing threshold was considered clinically relevant if there was an average loss of pure tone of at least $10 \mathrm{~dB}$ compared to the hearing threshold on audiograms taken before starting chemotherapy.

\section{Statistical Analysis}

Statistical analysis was performed using R 2.11.1 (R Foundation for Statistical Computing, Vienna, Austria). To compare patients' hearing function, the areas under the threshold curves (at 500-2000 and 6000$8000 \mathrm{~Hz}$ ) were calculated. The areas were tested for differences between subgroups using a Mann-Whitney $U$ test. The significance level was set at 0.005 .

\section{Results}

\section{Patients}

The age of the patients ranged from 32 to 71 years (mean $53.5 \pm 10.5$ years $) ; 61(70 \%)$ were older than 45 years. Of the 
patients, $73(83.9 \%)$ had undergone primary surgical treatment. The mean interval between surgery and first audiometric testing was $34.5 \pm 17.5$ days. All these patients received adjuvant chemotherapy. The remaining 14 (16.1\%) patients had only core biopsy for histological diagnosis before commencing primary chemotherapy. 11 (13\%) patients had a history of prior chemotherapy for other malignancies. No patients had any relevant associated co-morbidities, such as rhinosinusitis, otitis media, liver or renal problems, hyperactivity or hypoactivity of the thyroid gland, diabetes mellitus, high blood pressure or neurological disorders. The mean height of patients was $165.4 \pm 5.0 \mathrm{~cm}$ and and the mean weight $67.2 \pm 11.4 \mathrm{~kg}$. The clinico-pathological characteristics and chemotherapy regimens received by the patients are shown in table 1.

Table 1. Clinico-pathological characteristics and chemotherapy regimen in the patients

\begin{tabular}{lcr}
\hline Characteristics & $\mathrm{n}$ & $\%$ \\
\hline Total number of patients & 87 & \\
Histological diagnosis & 69 & 79 \\
$\quad$ Breast cancer & 12 & 14 \\
Ovarian cancer & 4 & 5 \\
Cervical cancer & 1 & 1 \\
Endometrial cancer & 1 & 1 \\
Vulva cancer & 11 & 13 \\
Secondary malignancy & 4 & 6 \\
Breast cancer & 1 & 1 \\
Ovarian cancer & 1 & 1 \\
Cervical cancer & 1 & 1 \\
Endometrial cancer & 1 & 1 \\
Vulva cancer & 1 & 1 \\
Chronic lymphocytic leukaemia & 2 & 2 \\
Hodgkin disease & & \\
Chemotherapy regimen & 4 & 5 \\
Cyclophosphamide, methotrexate, fluorouracil & 20 & 23 \\
Anthracycline containing* & 39 & 45 \\
Anthracycline and taxane containing** & 5 & 6 \\
Cisplatin & 19 & 21 \\
Carboplatin +/- paclitaxel & \multicolumn{2}{|c}{} \\
\hline *Fluorouracil, epirubicin and cyclophosphamide. & \\
**Fluorouracil, epirubicin, and cyclophosphamide/docetaxel; & \\
docetaxel monotherapy; docetaxel, doxorubicin, and cyclophosphamide; \\
dose-dense epirubicin, paclitaxel, and cyclophosphamide; or epirubicin, \\
paclitaxel, cyclophosphamide, and capecitabine (German Adjuvant \\
Intergroup Node-Positive Study). \\
\hline
\end{tabular}

Table 2. The average score of the subjective assessments of the patients hearing function, tinnitus, vertigo and feeling of pressure within the ear on visual analogue scale before commencement and 3 months after completion of chemotherapy

\begin{tabular}{lrr}
\hline & Before chemotherapy & $\begin{array}{c}3 \text { months after } \\
\text { chemotherapy }\end{array}$ \\
\hline Hearing & $83.0 \pm 17.2$ & $84.8 \pm 16.9$ \\
Tinnitus & $10.5 \pm 25.4$ & $9.4 \pm 23.8$ \\
Vertigo & $4.9 \pm 12.4$ & $18.5 \pm 30.9$ \\
Ear pressure & $4.9 \pm 16.4$ & $7.1 \pm 19.2$ \\
\hline
\end{tabular}

\section{Subjective Assessment}

The subjective gradings (on a visual analogue score of 0-100) of hearing function, presence of tinnitus, vertigo and a feeling of ear pressure for the patients before chemotherapy and 3 months after completion are shown in table 2. The scores for hearing function or preservation and perception of noise in the ear (tinnitus) by the patients before and 3 months after chemotherapy were similar. However, a few patients experienced vertigo and perception of ear pressure 3 months after chemotherapy (table 2). Immediately after completion of chemotherapy, on the visual analogue scale score of $0-100$, only a few patients perceived reduction in their hearing (average $16.8 \pm 29.3)$, experienced new tinnitus $(9.4 \pm 23.8)$, vertigo $(18.5 \pm 30.9)$ or ear pressure $(7.1 \pm 19.2)$.

\section{Audiometric Results}

There were no clinically relevant differences of up to $10 \mathrm{~dB}$ loss, for any of the patients, between the hearing threshold before the start, during ( 9 weeks), immediately after (18 weeks), and up to 3 months after completion of chemotherapy (fig. 1). Comparing the hearing threshold of the patients receiving platinum analogue-based chemotherapy with those for patients receiving non-platinum analogue-based chemotherapy, no significant differences were found in hearing threshold at any time during the study period (table 3 ). Looking at the audiogram for cisplatin- and carboplatintreated patients, the hearing threshold had not worsened by 3 months after completion of treatment (fig. 2). In the nonplatinum-based group, there was also no observed diminished
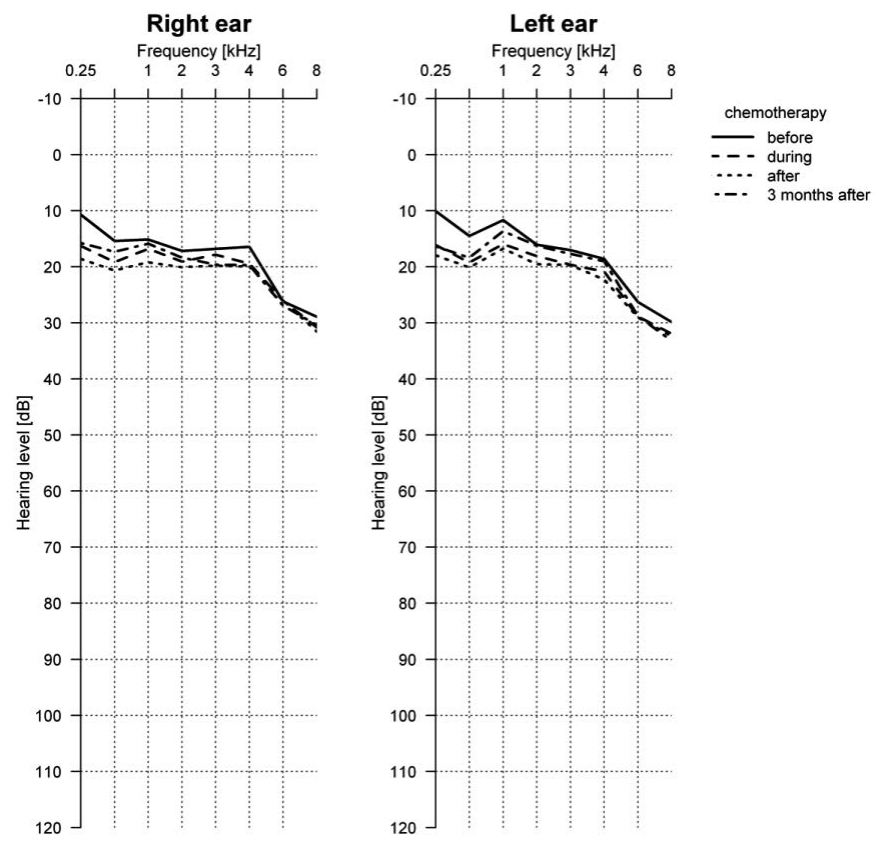

Fig. 1. Comparison of hearing thresholds of gynaecological patients who received chemotherapy before, during, immediately after, and 3 months after completion of chemotherapy. 
hearing threshold during or after chemotherapy (fig. 3). This was also the case for patients who had previous exposure to chemotherapy with no reduction in hearing threshold seen on the audiograms (table 3 ). No significant differences in the hearing threshold were found for the various tumour diagnoses (table 3). When the hearing thresholds obtained before chemotherapy for patients aged 45 years or younger were compared to those for patients older than 45 years, there was a significant difference in the hearing threshold at 6 and $8 \mathrm{KHz}$, and this difference was observed in the subsequent audiometric tests. The older patients had worse hearing threshold for the high frequencies (table 3). However, the comparison of hearing thresholds of patients (old or young group) at 9 weeks, 18 weeks or 3 months after completion of chemotherapy did not show any significant differences.
Fig. 2. Comparison of hearing thresholds between gynaecological patients who had received cisplatin and carboplatin chemotherapy.

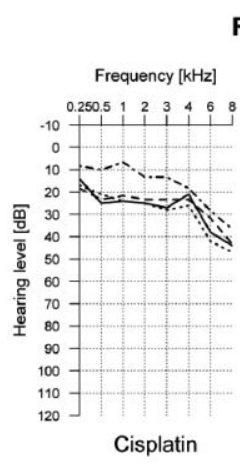

Right ear
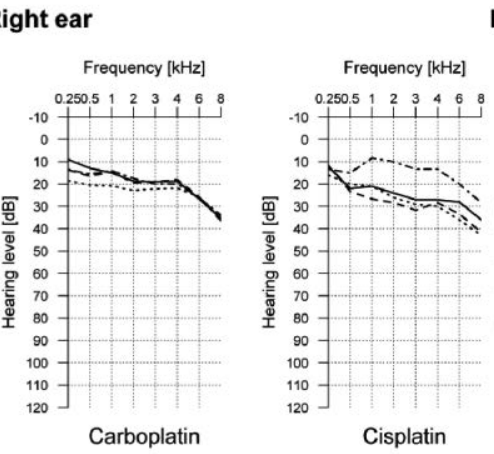

Left ear

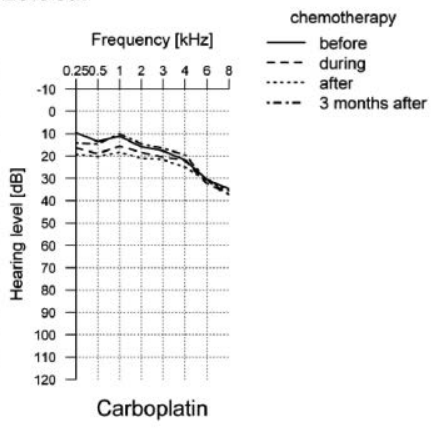

Table 3. Comparison of patients' hearing function from calculations of the areas under the threshold curves (500-2000 and 6000$8000 \mathrm{~Hz}$ ). The areas were tested for differences between subgroups using Mann-Whitney $\mathrm{U}$ test. The significance level was set at 0.005 right ear

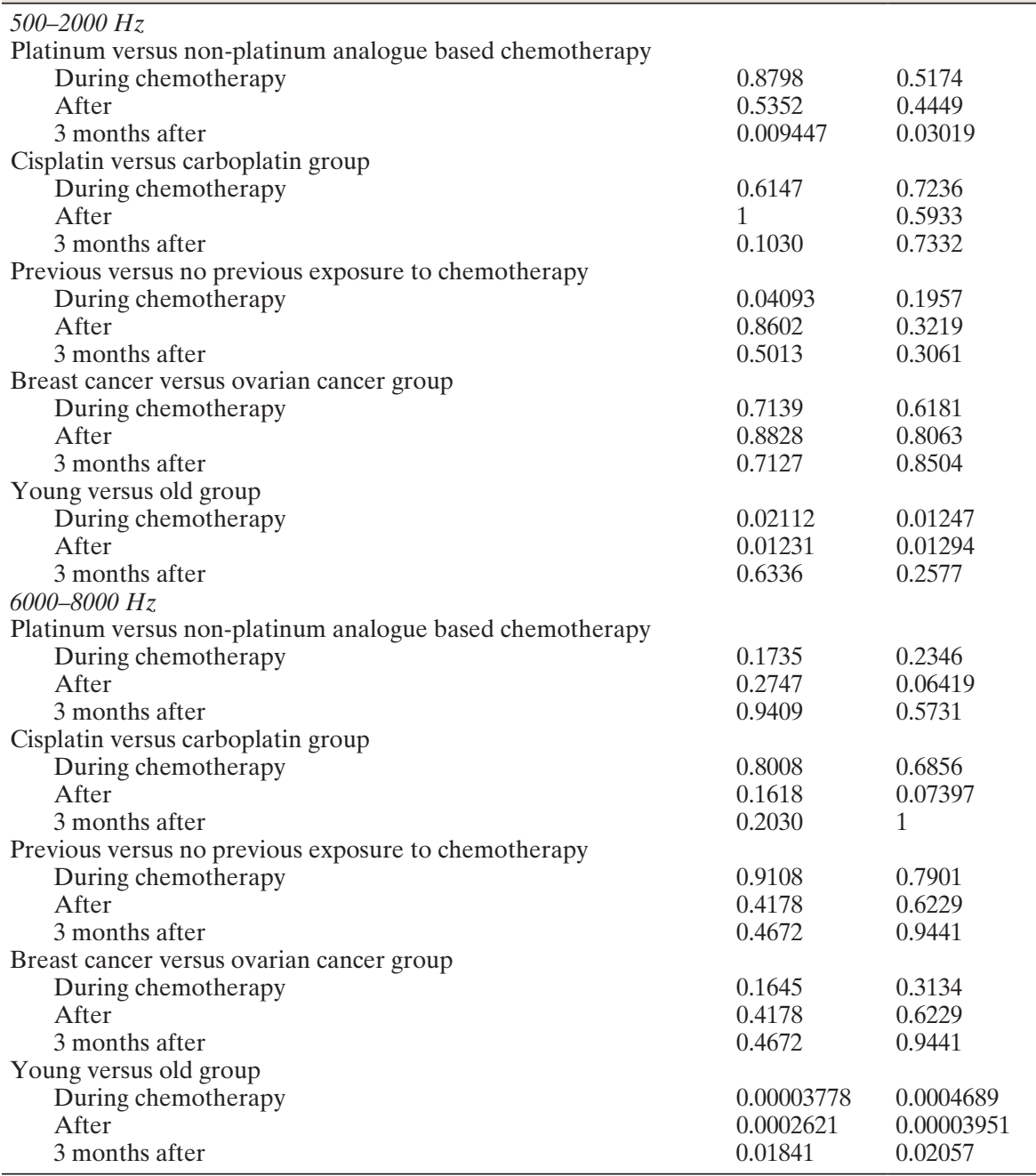

tinum versus non-platinum analogue based chemotherapy During chemotherapy 3 months after

During chemotherapy 3 months after During chemotherapy

3 months after

During chemotherapy

3 months after

$p$ value 


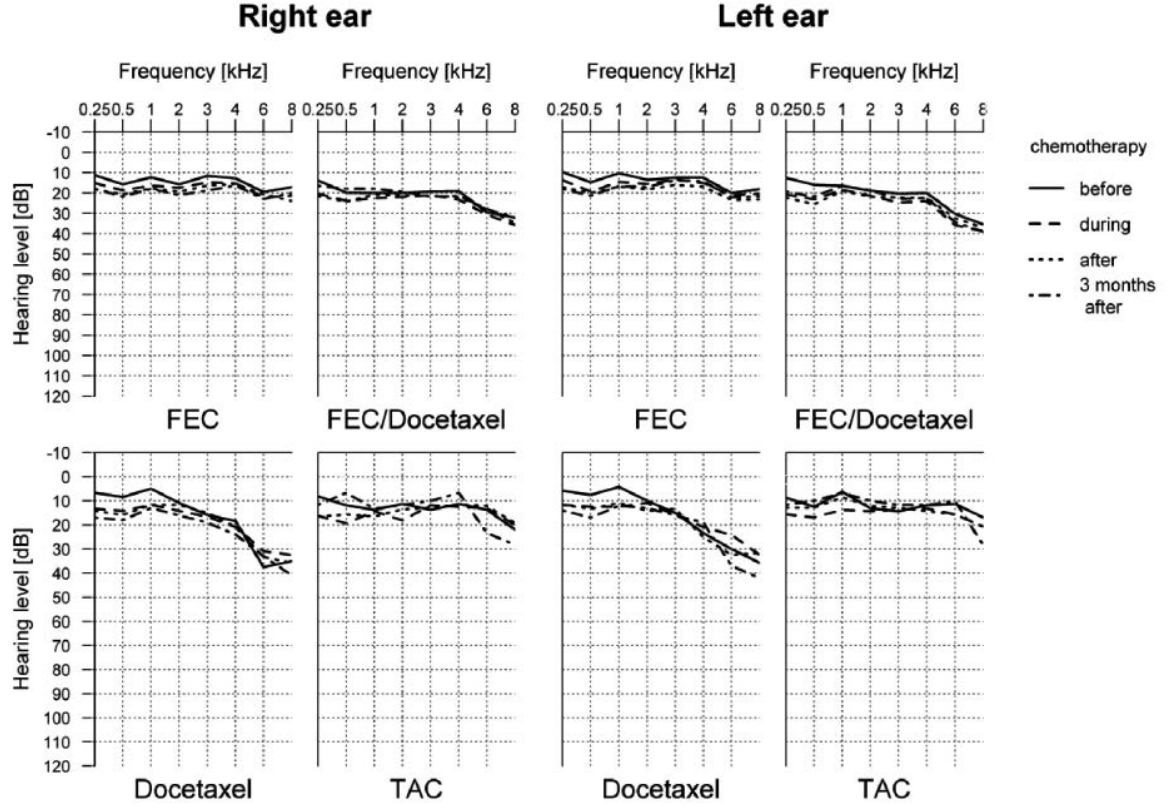

Fig. 3. Hearing thresholds of gynaecological patients who received non-platinum analoguebased chemotherapy.

\section{Discussion}

At 3 months after chemotherapy, the 87 women with gynaecological malignancies, who had been treated with chemotherapeutic agents, subjectively rated their hearing functions to be very good to excellent. To substantiate the subjective reports of the patients, the hearing function was tested with pure tone audiometry before, during, directly after, and 3 months after chemotherapy. No significant audiometric change in the speech hearing frequency $(0.5-2 \mathrm{KHz})$ was observed. This study showed that speech hearing frequencies were not significantly affected by the chemotherapeutic agents in the gynaecological patients followed in this study. This finding is in agreement with that of Jillella et al. [10], who did not observe clinical evidence of hearing impairment in any breast cancer patients treated with chemotherapy (cyclophosphamide, thiotepa and carboplatin). Although only $27 \%$ of the patients in this study received platinum-based chemotherapy, our findings also concurred with those of Laurell et al. [11], who did not observe a single case of significant change in the hearing frequency range of $0.5-2 \mathrm{KHz}$ in a larger gynaecological cancer population treated with cisplatin. Waters et al. [4] reported that the ototoxic effects of cisplatin, which included tinnitus and hearing loss in the speech frequency range, were associated with the administration of high dosages over a short period of time. Low-dose short-treatment regimens with either monthly administration of cisplatin or administration in block have been demonstrated to have low ototoxicity $[4,11]$. The ototoxicity induced by carboplatin has been demonstrated to be roughly similar to that of cisplatin when used in high dose $[6,7]$. The toxicity of carboplatin is dose limiting. Although the conventional dose of $350-400 \mathrm{mg} / \mathrm{m}^{2}$ per body surface area every 4 weeks is usually given, the recommendation for carboplatin administration, according to the Calvert formula, is: target AUC $\times(G F R+25)$. In this study the patients treated with platinum-based chemotherapy received either $40-50 \mathrm{mg} / \mathrm{m}^{2}$ of cisplatin or AUC5 of carboplatin and this might have accounted for the audiometric findings in these patients.

Hearing loss for high frequencies has been associated with high-dose platinum-based chemotherapeutic agents [11, 15]. Laurell et al. [11] reported high-frequency hearing loss in $22 \%$ of their patients in their older age group but did not find pretreatment with chemotherapy as a predictor for ototoxic change. Cisplatin ototoxicity has been demonstrated to cause an initial high-frequency hearing loss and the continued use of the drug has been shown to result in low-frequency hearing loss [16, 17]. High-frequency hearing loss can impact negatively on educational achievement, socio-emotional development, and quality of life. It was observed in this study that patients older than 45 years had a significantly high-frequency ( 6 and $8 \mathrm{KHz}$ ) hearing loss. This was observed in the audiometric tests of most of these patients before commencement, during, immediately after and 3 months after completion of the chemotherapy. Presbyacusis (high-frequency hearing loss) commonly occurs in older patients [18]. We did not find that chemotherapy led to a significant difference in the hearing threshold between older and younger groups.

Only 11 patients $(13 \%)$ in this study had history of prior chemotherapy for other malignancies. For these patients, no significant difference in their hearing threshold before, during and after completion of chemotherapy was found when compared to patients without a history of previous chemotherapy.

In this study $23 \%$ of the patients received an FEC (fluorouracil, epirubicin and cyclophosphamide) regimen, either alone or in combination with anthracycline, but no audiometric 
changes at either speech frequencies or high frequencies were observed. In contrast, Jenkins et al. [13] reported atypical hearing loss in 9 of 16 women $(64 \%)$ with breast cancer treated with FEC chemotherapy regimen. However, there was no clear statement on whether this observation had to do with FEC. Taxanes are new groups of anti-neoplastic agents used for chemotherapy and $45 \%$ of the patients in this study were treated with this medication. No significant audiometric changes were observed at either speech or high frequencies on the audiogram of these patients throughout the period of the study. This finding is in accordance with the work by Sarafraz et al. [12], who reported no audiovestibular side effects in 103 patients with either breast or ovarian cancers treated with taxanes during, and immediately after completion of, chemotherapy. There was also no difference at 3 months after completion of chemotherapy in this study.

The ototoxicity effects of chemotherapy are reflected not only in hearing loss but also in the clinical manifestations of sensory cochlea-vestibular complaints of tinnitus and vertigo. Although the subjective assessment in this study showed that the patients had no change in their hearing or in tinnitus 3 months after completion of chemotherapy, vertigo was experienced in more of these patients with $18.5 \pm 30.9$ on a visual analogue scale compared to $4.9 \pm 12.4$ before chemotherapy $(0=$ no vertigo, $100=$ strong vertigo $)$ as shown in table 2. This supports the evidence that chemotherapeutic agents may also have a toxic effect on the vestibular system. Benign paroxysmal positional nystagmus (BPPN) is the most common cause of vertigo in the general population and
Black et al. [19] have demonstrated an increased incidence of BPPN in patients receiving ototoxic medications. Although this study's primary interest was on hearing, this observed finding of subjective vertigo in some patients in this study would be an area to investigate further in future.

Al-Khatib et al. [20] in their study of children treated with platinum chemotherapy have reported the importance of long-term follow-up of these patients because ototoxicity can newly present or become worse years after completion of the therapy. Routine monitoring of patients on chemotherapy for early detection of signs of ototoxicity and initiation of preventive measures were suggested methods in patients being treated with ototoxic agent. However, the present study has shown that, in gynaecological patients, routine monitoring of hearing of cancer patients after each cycle of chemotherapy received is unnecessary up to a period of 3 months after completion of chemotherapy. This would help to minimise patients' financial commitments and frequent hospital visits.

In conclusion, deterioration of hearing function is uncommon in gynaecological patients treated with either platinumbased or non-platinum-based chemotherapy. Hence, routine audiometric test in these patients up to 3 months after completion of chemotherapy is not necessary.

\section{Disclosure Statement}

The authors have no conflicts of interest to declare.

\section{References}

1 Jemal A, Siegel R, Ward E, Hao Y, Xu J, Thun MJ: Cancer Statistics. CA Cancer J Clin 2009;59:225-249.

2 Karam AK: Breast cancer posttreatment surveillance: Diagnosis and management of recurrent disease. Clin Obstet Gynecol 2011; 54:157-163.

3 Katalinic A, Pritzkuleit R, Waldmann A: Recent trends in breast cancer incidence and mortality in Germany. Breast care 2009;4:75-80.

4 Waters GS, Ahmad M, Katsarkas A, Stanimir G, McKay J: Ototoxicity due to cis-diamminedichloroplatinum in the treatment of ovarian cancer: Influence of dosage and schedule of administration. Ear Hear 1991;12:91-102.

5 Rademaker-Lakhai JM, Crul M, Zuur L, Baas P, Beijnen JH, Simis YJ, van Zandwijk N, Schellens $\mathrm{JH}$ : Relationship between cisplatin administration and the development of ototoxicity. J Clin Oncol 2006;24:918-924.

6 Wake M, Takeno S, Ibrahim D, Harrison R, Mount R: Carboplatin ototoxicity: An animal model. J Laryngol Otol 1993;107:585-589.

7 Van Warmerdam LJ, Rodenhuis S, van der Wall E, Maes RA, Beijnen JH: Pharmacokinetics and pharmacodynamics of carboplatin administered in a high-dose combination regimen with thio- tepa, cyclophosphamide and peripheral stem cell support. Br J Cancer 1996;73:979-984.

8 Qie WX: Experimental study on ototoxicity of cisplatin. Zhonghua Er Bi Yan Hou Ke Za Zhi 1990;25:195-198.

9 Laurell G, Borg E: Cis-platin ototoxicity in previously noise-exposed guinea pigs. Acta Otolaryngol 1986;101:66-74.

10 Jillella AP, Britt GW, Litaker MS, Kallab AM, Harkness K, Garner GD: Ototoxicity after highdose chemotherapy with cyclophosphamide, thiotepa and carboplatin followed by stem cell transplantation in patients with breast cancer. Med Oncol 2000;17:287-292.

11 Laurell G, Borg E: Ototoxicity of cisplatin in gynaecological cancer patients. Scand Audiol 1988;17:241-247.

12 Sarafraz M, Ahmadi K: Paraclinical evaluation of side effects of taxanes on auditory system. Acta Otorhinolaryngol Ital 2008;28:239-242.

13 Jenkins V, Beveridge H, Low R, Mitra S: Atypical hearing loss in women with breast cancer receiving adjuvant treatment. Breast 2006;15:448-451.

14 Jenkins V, Low R, Mitra S: Hearing sensitivity in women following chemotherapy treatment of breast cancer: result from a pilot study. Breast 2009;18:279-283.
15 Huitema AD, Spaander M, Mathjt RA, Tibben MM, Holtkamp MJ, Beijnen JH, Rodenhuis S: Relationship between exposure and toxicity in high-dose chemotherapy with cyclophosphamide, thiotepa and carboplatin. Ann Oncol 2002;13:374 384.

16 Zuur CL, Simis YJ, Landsdaal PE, Rasch CR, Tange RA, Balm AJ, Dreschler WA: Audiometric patterns in ototoxicity of intra-arterial cisplatin chemoradiation in patients with locally advanced head and neck cancer. Audiol Neurootol 2006; 11:318-330.

17 Schultz C, Goffi-Gomez MV, Liberman PH, Carvalho AL: Report on hearing loss in oncology. Braz J Otorhinolaryngol 2009;75:634-641.

18 Sprinzl GM, Riechelmann H: Current trends in treating hearing loss in elderly people: a review of the technology and treatment options - a minireview. Gerontology 2010;56:351-358.

19 Black FO, Pesznecker SC, Homer L, Stallings V: Benign paroxysmal positional nystagmus in hospitalized subjects receiving ototoxic medications. Otol Neurotol 2004;25:353-358.

20 Al-Khatib T, Cohen N, Carret AS, Daniel S: Cisplatinum ototoxicity in children, long-term follow up. Int J Pediatr Otorhinolaryngol 2010;74: 913-919. 